\title{
Using Transcranial Magnetic Stimulation (TMS) to Compare Cortical Excitability Among Depressed Patients, Sleep-Deprived Controls, and Rested Controls
}

\author{
Rajani Rajan \\ Case Western Reserve University \\ Paul A. Zarkowski \\ University of Washington School of Medicine; Case Western Reserve University \\ Pedro L. Delgado \\ Case Western Reserve University; University Hospitals of Cleveland
}

Follow this and additional works at: https://jdc.jefferson.edu/jeffjpsychiatry

Part of the Psychiatry Commons

Let us know how access to this document benefits you

\section{Recommended Citation}

Rajan, Rajani; Zarkowski, Paul A.; and Delgado, Pedro L. (2005) "Using Transcranial Magnetic Stimulation (TMS) to Compare Cortical Excitability Among Depressed Patients, Sleep-Deprived Controls, and Rested Controls," Jefferson Journal of Psychiatry. Vol. 19 : Iss. 1 , Article 5.

DOI: https://doi.org/10.29046/JJP.019.1.004

Available at: https://jdc.jefferson.edu/jeffjpsychiatry/vol19/iss1/5

This Article is brought to you for free and open access by the Jefferson Digital Commons. The Jefferson Digital Commons is a service of Thomas Jefferson University's Center for Teaching and Learning (CTL). The Commons is a showcase for Jefferson books and journals, peer-reviewed scholarly publications, unique historical collections from the University archives, and teaching tools. The Jefferson Digital Commons allows researchers and interested readers anywhere in the world to learn about and keep up to date with Jefferson scholarship. This article has been accepted for inclusion in Jefferson Journal of Psychiatry by an authorized administrator of the Jefferson Digital Commons. For more information, please contact: JeffersonDigitalCommons@jefferson.edu. 


\title{
Using Transcranial Magnetic Stimulation (TMS) to Compare Cortical Excitability Among Depressed Patients, Sleep-Deprived Controls, and Rested Controls
}

\author{
Rajani Rajana, Paul Zarkowski M.D. ${ }^{\text {b }}$, and Pedro L. Delgado, M.D.c
}

\begin{abstract}
OBJECTIVE: To compare cortical excitability among sleep-deprived and rested controls, and depressed subjects. METHODS: 3 controls and 4 depressed patients (average HAM-D 19) were recruited. Sleep-deprived controls were instructed to sleep $<=4$ hours the previous night. TMS-produced motor evoked potentials (MEPS) were measured before and after subjects engaged in sets of non-fatiguing manual exercise. RESULTS: Post-exercise MEP increased $71+1-19 \%$ for rested controls, $28+1-32 \%$ for sleep deprived controls, and $8+1-15 \%$ for depressed patients. This increase, "facilitation," was significantly greater in rested controls than in depressed patients $(p=0.011)$. No significant difference in facilitation was found between sleep-deprived controls and depressed patients. CONCLUSION: Sleep-deprivation may partially account for reduced cortical excitability in depressed patients.
\end{abstract}

Key Words: transcranial magnetic stimulation, TMS, cortical excitability, sleep deprivation

\section{INTRODUCTION}

Transcranial magnetic stimulation (TMS) is rapidly gaining prominence as a new therapeutic instrument for the treatment of patients with psychiatric illnesses. However, there is also a growing body of research in which TMS has been used as a tool to measure such neurophysiological parameters as cortical excitability in mental illness and health.

Magnetic stimulation of the brain involves the non-invasive induction of electrical currents in neurons, via the external application of a magnetic field. Typically, the source of the field is either a circular "doughnutshaped" coil or figure-of-eight "butterfly" coil. In either case, the magnetic field generated is focal, with a maximal resolution of $0.5 \mathrm{~cm}$. When laid gently on the subject's head, the focal magnetic field induces circular electrical currents in the brain, which in turn depolarizes cortical interneurons. Consequently, when the primary motor cortex is stimulated, the corresponding muscle groups contract. In contrast to electric fields, magnetic fields are not affected by impedance effects due to the scalp and skull. The field typically reaches to a depth of $2 \mathrm{~cm}$ below the scalp-approximately reaching to the level of the gray-white matter junction of the cerebral cortex (1). The technique is noninvasive, relatively painless, and has been carefully studied in several safety trials (2).

\footnotetext{
a Rajani Rajan is a graduate of Case Western Reserve University School of Medicine in Cleveland, OH. At the time of the preparation of this study, she was an MSIV at Case Western Reserve University. She was supported by the American Psychiatric Association's Program for Minority Research Training in Psychiatry.

b Paul Zarkowski is currently an acting assistant professor at the University of Washington School of Medicine, Department of Psychiatry in Seattle, Washington. At the time of the preparation of this study, he was an EEG fellow at Case Western Reserve University Department of Psychiatry.

c Pedro Delgado is the chairman of the Psychiatry Department at Case Western Reserve University/University Hospitals of Cleveland in Cleveland, OH. Correspondence to: Rajanirajan2@aol.com (email address may be published) 349 Stanford Ct. Irvine, CA 92612
} 
The TMS field is focal in breadth and depth. For this reason, the technique is ideal for studying the specific, limited effects of local stimulation of cortical tissue. In particular, cortical excitability of particular portions of the primary motor cortex may be measured by determining the effect of stimulation on specific corresponding muscle groups. Typical muscle groups used to assess cortical excitability are the first dorsal interosseous muscle of the hand, the extensor carpi radialis of the arm, and the abductor pollicis brevis (APB) found in the thumb. Electromyography (EMG) can be used to record potentials that are evoked in the muscle after the stimulation of the corresponding area of the motor cortex. These potentials are known as "motor evoked potentials" or "MEPs." The effect of stimulation may also be observed by a visible twitch in the muscle.

Post-exercise facilitation studies typically observe changes in TMS MEP production before and after either fatiguing or non-fatiguing exercise. In these studies, there is a baseline measurement of MEP amplitude of the muscle group stimulated at the cortex. Then, the muscle group to be studied is subjected to either fatiguing or non-fatiguing voluntary exercise, after which there is a repeat stimulation of the motor cortex and a measurement of the amplitude of the MEP seen on EMG ("MEP amplitude"). Previous studies have shown that after nonfatiguing exercise, healthy patients typically experience "facilitation." Facilitation is measured by a relative increase in MEP amplitude after exercise. This effect is thought to be mediated primarily by intracortical, interneuronal mechanisms, as opposed to a purely spinal or peripheral muscular effect $(4,5)$. Indeed, recent studies show no evidence of post-exercise MEP changes after peripheral TMS stimulation at the wrist (3).

Several recent studies have measured cortical excitability using TMS and EMG in patients with psychiatric illnesses. In one study, 11 patients with schizophrenia and 10 patients with major depression demonstrated decreased post-exercise facilitation in comparison with a group of 13 healthy control patients (7). Another study involving 14 patients with mania, 14 with major depression, 14 with schizophrenia, and 14 healthy controls were studied and in each non-control group, subjects showed significantly reduced post-exercise facilitation (8).

A study by Samii et al involving 18 control subjects, 12 subjects with chronic fatigue syndrome, and 10 depressed patients showed significantly reduced post-exercise facilitation after nonfatiguing exercise in depressed patients, and in patients with chronic fatigue syndrome (9). In this study, mean MEP amplitude noted after nonfatiguing exercise was $218 \%$ of the baseline MEP amplitude $(p=0.0002)$, compared with $126 \%(p=0.023)$ in chronic fatigue patients, and $155 \%(p=0.059)$ in depressed patients. In all of these subjects, there was an increase in MEP amplitude, facilitation, after exercise as compared with the resting state. However, this facilitation was noted to be less in patients with either depression or chronic fatigue, and this effect was statistically significant $(\mathrm{p}=0.003)$ for chronic fatigue and depressed patients $(\mathrm{p}=.039)$, although there was no statistically significant difference in facilitation between depressed patients and patients with chronic fatigue syndrome. Baseline MEP values prior to exercise were not statistically different across all groups studied. A similar study by Shajahan et al in which post-exercise facilitation was studied in 10 depressed patients, 10 healthy controls, and 10 patients in recovery from depression also demonstrated statistically significant decrease in facilitation in depression as compared to either group $(\mathrm{p}=0.005$ and $\mathrm{p}=$ 0.012 respectively). Again, baseline MEP values were not statistically different across all groups (5).

Patients with depression as well as those with other psychiatric disorders often report poor sleep as part of their illness. For this reason, we were interested in the question of whether poor sleep alone might explain the decreased cortical excitability seen in patients with depression and other psychiatric disorders. Previous studies have analyzed the effect of sleep-deprivation on cortical excitability using the "paired-pulse" technique. This technique, in which MEPs are measured subsequent to a higher-intensity pulse that follows milliseconds after a lower intensity conditioning pulse, has been used to study different parameters of cortical excitability. If the MEP produced after the second pulse results in an increase in MEP amplitude, this is considered intracortical "facilitation." If the MEP produced after the second pulse results in a decrease in MEP 
amplitude, this is considered intracortical inhibition. An interstimulus interval of 2-3 ms has been shown to create intracortical inhibition. An interstimulus interval of $14-16 \mathrm{~ms}$ has been shown to create intracortical facilitation.

A paired-pulse study of cortical excitability by Manganotti et al (10), has reported that sleep deprivation in healthy subjects significantly increases motor threshold. Motor threshold is the minimum intensity of a single pulse of magnetic stimulation required to produce an evoked potential in a target muscle. In addition, this study also found increased intracortical inhibition during maximal sleepiness in these subjects. The study also found a trend of decreased facilitation in sleep-deprived patients; however this trend was not statistically significant. The authors of this study concluded that their findings were suggestive of decreased cortical excitability in healthy sleep-deprived patients.

A paired-pulse study by Civardi et al studied eight healthy sleep-deprived subjects, and found no significant change in motor threshold across the groups (6). This study did, however, show a statistically significant reduction in both paired-pulse inhibition and facilitation in the sleep-deprived subjects. Neither Civardi nor Manganotti studied cortical excitability using the method of post-exercise facilitation. Studies using postexercise facilitation in particular have shown the most consistent changes in cortical excitability in mentally ill patients.

Here, we report the outcome of a TMS study that compares post-exercise facilitation among depressed patients, sleep-deprived controls, and rested controls. The objective was to determine if sleep deprivation in control subjects had an effect on cortical excitability (specifically, post-exercise facilitation) and to compare this effect with those effects observed in rested controls and in depressed patients. The post-exercise facilitation method of assessing cortical excitability used in several studies of mentally ill patients, has not been studied in healthy, sleep deprived controls. We hypothesized that sleep-deprived healthy controls would have significantly diminished post-exercise facilitation compared to rested controls. Secondly, we hypothesized that depressed patients would have significantly diminished facilitation compared to healthy, rested controls. Finally, we hypothesized there would be no statistically significant difference in post-exercise facilitation between depressed and healthy sleep-deprived controls.

\section{METHODS}

Seven right-handed, female subjects were recruited for this study. The 3 control subjects had a mean age of 28 years (range 23-34). These controls were used in both the rested and sleep-deprived arms of the study. Controls were screened and excluded for any current or previous history of substance abuse/dependence or mental illness. None of the control subjects were taking or had recently taken psychotropic medications. The 4 depressed patients all had a current diagnosis of Major Depressive Disorder by DSM-IV TM criteria. Depressed patients had a mean age of 32 years. The Hamilton Depression Scale was administered to these patients and the average score was 19 (range 16-23). Psychotropic medications currently or previously used in these patients included paroxetine, citalopram, olanzapine, risperidone, and zolpidem. One subject was currently using birth control pills.

All subjects were given a neurological and psychiatric examination (which included the Hamilton Depression Scale). Subjects were excluded for any history of major medical illness, any neurological illness (including epilepsy, seizures, increased intracranial pressure), any presence of metal in the cranium, any signs of major medical or neurological illness on examination, any family history of epilepsy, or age under 18 years or over 80 years.

The measurement of post-exercise facilitation was repeated in the control group after a night of poor sleep. These controls were asked to return at least 3 weeks after the initial "rested" portion of the TMS procedure. 
Sleep deprived controls were instructed to sleep for no more than 4 hours the previous night, while depressed patients and rested controls were permitted to sleep unrestrictedly the previous night. None of the control subjects consumed caffeine within 24 hours of the procedure. Rested controls did not report any changes in normal baseline sleep, and reported feeling rested, awake, and alert. Sleep-deprived controls reported feeling tired compared to their self-reported baseline, but were awake, alert, and able to understand and follow instructions.

TMS procedure and post-exercise facilitation experiment was based on a procedure similar to that outlined in Shajahan et al 1999 (5). Patients were seated in a comfortable chair with forearms supported by an armrest and their forearm and palm in a supine position. Maximal pinch strength was measured using a pinch gauge dynamometer (B\&L Engineering, Costa Mesa, California, USA). A Magstim 200 TMS (Magstim Co. Limited, Whitland, Dyfed, UK) was used for the TMS procedure, using a $70 \mathrm{~mm}$ "figure of eight" coil. Subjects were instructed to keep their hands as still and relaxed as possible during MEP recording. Evoked potentials/MEPs were recorded from noninvasive surface electrodes, which were attached to the APB of the right hand. A non-abrasive conductive electrode cream was used to ensure artifact-free recording of the MEP. MEPs were recorded using a Dantec Neuromatic 2000 M EMG (Dantec Electronics Limited, Bristol, $\mathrm{UK})$.

Then, motor threshold associated with the abductor pollicis brevis area of the motor cortex was found in all subjects. Motor threshold was determined first by slowly increasing the magnitude of the magnetic stimuli over the left motor cortex until a MEP of at least 50 microvolts was measured over the right APB in 5 out of 10 consecutive trials. Evoked potentials were measured on the EMG. After a 30 minute rest period, baseline measurements of MEPs were administered at $0.25 \mathrm{~Hz}$, at a magnitude of $115 \%$ of the motor threshold stimulus intensity, in trains of 5 pulses with each train separated by 30 seconds. Ten such trains were administered for a total of 50 stimuli.

Next, subjects exercised the APB for 15 seconds at $20 \%$ of their maximal strength (nonfatiguing exercise). Subsequently, they were magnetically stimulated at $115 \%$ of their motor threshold stimulus intensity over the same spot over the motor cortex for a train of 5 stimuli at $0.25 \mathrm{~Hz}$. 5 exercise-stimulation sets were administered.

All patients were given a detailed explanation of the procedure and all patients signed a written informed consent statement. This protocol (05-02-36J) was approved by the Institutional Review Board for Human Investigation at University Hospitals of Cleveland.

\section{RESULTS}

Statistical analysis was conducted using the NCSS-PASS software package for Windows. The mean baseline motor evoked potential and mean percent increase in MEP after exercise (post-exercise facilitation) are shown in Table 1 for each group. The amount of post-exercise facilitation was found to be significantly decreased $(\mathrm{p}=0.011$ unequal variance $\mathrm{t}$-test) in the depressed group compared to the group of rested healthy controls. MEP for rested controls increased $71+/-19 \%$ after exercise compared to baseline while depressed patients' post-exercise MEP increased only $8+/-15 \%$. Sleep-deprived controls had a MEP increase of 28 $+/-32 \%$. The sleep-deprived control group showed a decreased post-exercise facilitation that was not significantly different from that of the depressed patient group ( $\mathrm{p}=0.30$, unequal-variance t-test). No significant difference was seen in baseline MEPs across all groups ( $\mathrm{F}=0.93$, ANOVA). The post-exercise facilitation in the control group was decreased after a night of sleep deprivation compared to that observed in the rested state, but this trend was not significant ( $\mathrm{p}=0.25$, Hotelling's one sample t-test). 
Table 1

\begin{tabular}{|lll|}
\hline Baseline MEP (mV) & Post-exercise Facilitation (\%) \\
\hline Depressed Patients & $0.76(0.44)$ & $9(15)$ \\
\hline Rested Controls & $0.60(0.86)$ & $71(19)$ \\
\hline Sleep-deprived Controls & $0.99(0.82)$ & $28(32)$ \\
\hline
\end{tabular}

The baseline motor evoked potential (MEP) and percent post-exercise facilitation by group. Values are mean (standard deviation). All motor evoked potentials are measured with TMS intensity set at $115 \%$ of motor threshold.

\section{DISCUSSION}

In agreement with Samii et al, and Shajahan et al, we found a statistically significant reduction in post-exercise facilitation in depressed patients compared to healthy rested controls. As mentioned earlier, decreased post-exercise facilitation has been understood to reflect reduced excitability at the cortical level in these patients.

Importantly, our study found no statistically significant difference in post-exercise facilitation between depressed patients and sleep-deprived controls. This finding is significant in light of the prevalence of disturbed sleep among patients with mental illness. The reduced post-exercise facilitation reported in patients with mental disorders may reflect an intracortical disturbance due to sleep-deprivation instead of psychiatric pathology.

While both sleep-deprived and depressed patients exhibited a decrease in post-exercise facilitation compared to healthy controls, the decrease was a non-significant trend in the sleep-deprived population. We suspect that the more pronounced decrease in facilitation found in depressed patients may be attributable to greater and more prolonged sleep disturbance in psychiatrically depressed patients. We also acknowledge the contribution of other factors intrinsic to the underlying mechanisms of depression itself (sleep-cycle differences), and sample size effects.

Both the Civardi and Manganotti studies showed statistically significant changes in cortical excitability after sleep-deprivation; however these studies employed a different measure of cortical excitability, which may affect different intracortical mechanisms. Also, these studies did not employ the technique of post-exercise facilitation, which has shown the most consistent findings in patients with mental illness.

Additional cortical excitability studies, with larger sample sizes, may help further elucidate the role sleep deprivation per se may play in cortical excitability in patients with psychiatric illnesses and perhaps in the psychomotor retardation observed in depressed patients. Studies correlating specific sleep cycle disturbances with cortical excitability in mentally patients with psychiatric illnesses may help define the contribution of sleep disturbances to psychomotor symptomatology. Nonetheless, we believe our study is suggestive that poor sleep may be a factor to control for in future studies of cortical excitability in psychiatric patients and healthy controls.

\section{ACKNOWLEDGEMENTS}

We wish to thank B\&L Engineering for the repair and calibration of the pinch gauge dynamometer. 


\section{REFERENCES}

1. Lisanby SH, Luber B, Perera T, et al. Transcranial Magnetic Stimulation: applications in basic neuroscience and neuropsychopharmacology. Int J of Neuropsychopharmacology 2000; 3:259-273.

2. Green RM, Pascual-Leone A, Wasserman EM. Ethical Guidelines for rTMS research. IRB 1997; MarApr 19 (2): 1-7.

3. Zanette G, Bonato C, Polo A, et al. Long-lasting depression of motor-evoked potentials to transcranial magnetic stimulation following exercise. Exp Brain Res 1995; 107 (1): 80-6.

4. Bonato $\mathrm{C}$, Zanette $\mathrm{G}$, Polo A, et al. Cortical output modulation after rapid repetitive movements. Ital J Neurol Sci 1994; Dec 15 (9): 489-94.

5. Shajahan PM, Glabus MF, Jenkins JA, et. al.. Post-exercise motor evoked potentials in depressed patients, recovered depressed patients and controls. Neurology 1999; 53: 644-6.

6. Civardi C, Boccagni C, Vicentini R, Cortical excitability and sleep deprivation: a transcranial magnetic stimulation study. J. Neurol Neurosurg Psychiatry 2001; 71: 209-12.

7. Reid PD, Daniels B, Rybak M, et al. Cortical excitability of psychiatric disorders: reduced post exercise facilitation in depression compared to schizophrenic and controls. Aus N Z J Psychiatry 2002; 36: 66973.

8. Chroni E, Lekka NP, Beratis S, et al. Effect of exercise on motor evoked potentials elicited by transcranial magnetic stimulation in psychiatric patients. J Clin Neurophysiol 2002; 19: 240-4.

9. Samii A, Wassermann EM, Ikoma K, et al. Decreased postexercise facilitation of motor evoked potentials in patients with chronic fatigue syndrome or depression. Neurology 1996; 47: 1410-4.

10. Manganotti P, Palermo A, Patuzzo S, Zanette G, Fiashci A. Decrease in motor excitability in human subjects after sleep deprivation. Neurosci Lett 2001; 304: 153-6. 